\title{
Error tracking in a clinical biochemistry laboratory
}

\author{
Pal Bela Szecsi ${ }^{1,2, *}$ and Lars Ødum ${ }^{1}$ \\ ${ }^{1}$ Department of Clinical Biochemistry, University of \\ Copenhagen, Roskilde Hospital, Roskilde, Denmark \\ ${ }^{2}$ Department of Clinical Biochemistry, University of \\ Copenhagen, Gentofte Hospital, Hellerup, Denmark
}

\section{Abstract \\ Background: We report our results for the systematic recording of all errors in a standard clinical laboratory over a 1-year period.}

Methods: Recording was performed using a commercial database program. All individuals in the laboratory were allowed to report errors. The testing processes were classified according to function, and errors were classified as pre-analytical, analytical, post-analytical, or service-related, and then further divided into descriptive subgroups. Samples were taken from hospital wards $(38.6 \%)$, outpatient clinics $(25.7 \%)$, general practitioners $(29.4 \%)$, and other hospitals.

Results: A total of 1189 errors were reported in 1151 reports during the first year, corresponding to an error rate of 1 error for every 142 patients, or 1 per 1223 tests. The majority of events were due to human errors $(82.6 \%)$, and only a few $(4.3 \%)$ were the result of technical errors. Most of the errors $(81 \%)$ were preanalytical. Of the remainder, $10 \%$ were analytical, $8 \%$ were post-analytical, and $1 \%$ was service-related. Nearly half of the errors $(n=550)$ occurred with samples received from general practitioners or clinical hospital wards. Identification errors were relatively common when non-technicians collected blood samples.

Conclusions: Each clinical laboratory should record errors in a structured manner. A relation database is a useful tool for the recording and extraction of data, as the database can be structured to reflect the workflow at each individual laboratory.

Clin Chem Lab Med 2009;47:1253-7.

Keywords: diagnostic errors; medical errors; safety management.

\section{Introduction}

In recent years, health care has increasingly focused on reducing errors and near-errors. Following a vol-

*Corresponding author: Pal B. Szecsi, Department of Clinical Biochemistry, University of Copenhagen, Gentofte Hospital, Niels Andersens Vej 65, 2900 Hellerup, Denmark Phone: + 453977 7494, Fax: + 453977 7616,

E-mail: palsze01@geh.regionh.dk

Received April 13, 2009; accepted June 28, 2009;

previously published online August 7, 2009 untary trial of error recording systems at hospitals, Denmark passed legislation in 2003 that required all health care personnel to report errors and near-errors (Law No. 429 of 10/06/2003). While all types of errors can be reported, the focus has been on errors related to medication, identification, and other serious errors occurring in clinical departments. Beginning January 1,2004 , event reports have been collected in a central database at the Danish National Institution of Health.

Hospital laboratories, even small departments, perform procedures numbering in the millions. Clinical biochemistry laboratories have focused on quality control and reducing error rates by introducing automation, standardisation, and electronic handling of test requests and test results. Despite these efforts, there are still numerous errors associated with clinical biochemistry laboratories.

The field was recently reviewed (1-4); while no Danish reports have been published regarding biochemical laboratories, studies in other countries have reported error rates of $0.33 \%-0.47 \%$ for test results and $0.05 \%-0.11 \%$ for patients $(5)$. It is important to monitor the complete testing process, including the pre- and post-analytical phases, where many errors occur $(2,4,6)$.

Late in 2002, the Department of Clinical Biochemistry at Roskilde Hospital began recording all errors in order to monitor the types and frequency, with the aim of reducing the error rate. We report here our findings from the first complete year of the error database.

\section{Materials and methods}

The staff at the Department of Clinical Biochemistry, Roskilde Hospital (excluding the blood bank) consists of $\sim 85$ individuals, corresponding to 65 full-time positions. All of these employees are technicians, with the exception of two MDs, two pharmacists, and three secretaries. The laboratory staff performed nearly all phlebotomies and electrocardiograms at the hospital wards throughout the entire day, and in the laboratory phlebotomy outpatient clinic during the daytime. Similar services were also provided at a nearby psychiatric hospital, a phlebotomy clinic, and in the private homes of immobilised patients. Arterial blood samples and urine samples were collected and transported to the laboratory by the staff at the ward. At the hospital wards and outpatient clinics, samples were ordered electronically through the laboratory information system (LIS). At birth, all Danish citizens are provided a unique identification number, known as a centralpersonal-registration-number (CPR-NR). This 10-digit ID number identifies the person using birth date, gender, age, and a check cipher to verify the CPR-NR. The CPR-NR is used in the LIS to identify patients, and the LIS is linked to the national CPR-NR database to provide information about the patient, such as patient name and address. When samples 
are ordered electronically, a sample collection sheet with labels and a patient ID (CPR-NR) are generated within the laboratory. This sheet was used by the technicians when they collected samples. The technicians transported all samples to the laboratory. General practitioners ordered tests using a pre-printed paper form with labels during 2003 and 2004. The practitioners identified the patients and transferred the patient ID from their patient record system to these forms using either manual transcription or by electronic means. When the samples and forms arrived at the laboratory, these data were manually transcribed into the LIS. Some practitioners referred the patients for phlebotomy at the laboratory outpatient clinic; other practitioners collected, centrifuged, and transported or mailed the samples to the laboratory. These samples included blood and urine as well as cervical swab specimens for Chlamydia testing. Test results were instantly available at hospital wards through the LIS system or through a printed version that was mailed daily. Most results for general practitioners were transmitted electronically to the practitioners' individual electronic patient record system.

During 2003, tests were performed on 168,734 patients and included 1,454,251 analyses for more than 200 analytes. The test repertoire included basic chemical, haematological, microbiological, serological, and DNA tests. Samples were exchanged in collaboration with the sister department at the nearby Køge Hospital, other hospitals, and special laboratories. In total, 33,309 samples were sent to other laboratories. The majority of samples came from the hospital wards $(38.6 \%)$ and outpatient clinics (25.7\%). General practitioners accounted for $29.4 \%$ of the samples, and the remainder came from other hospitals.

An error made within the laboratory was defined as any aberration from routine procedures according to internal instructions or complaints concerning tests from wards or practitioners (7).

Errors made outside the laboratory were detected based on unclear or missing information, the ordering of inappropriate tests (e.g., a prostate-specific antigen test on a female patient), or discrepancies between the ordered test and delivered material. Errors related to research work were not included.

Initially, errors were reported on paper with a brief description of the event. These reports indicated whether the errors were human or technical in nature and included the severity of the error. We quickly realised that a more systematic approach was needed to deal with the large number of events, and to allow us to draw conclusions from the data. Therefore, we sought to implement a database with a search capability to record errors. In order to treat the data consistently and to build a useful database, it was necessary to analyse departmental functions in detail. Event recording was performed using a commercially available relational database program, Filemaker Pro (Santa Clara, CA, USA). This program runs on both Windows and Macintosh computers, and allows data to be transferred and shared over the Internet. All individuals in the laboratory were encouraged to report errors using a paper form with printed fields for types of errors and explanatory text. Reports were not anonymous in order that supplemental information could be ascertained. However, it was emphasised that no repercussions would result from filing of reports. Qualified technicians checked, archived, and entered the reports into the database.

All test procedures were divided into groups and subgroups as shown in Table $1(2,8,9)$. Only the system administrator (the corresponding author) was allowed to delete data or alter the database structure. The data were exported monthly into an Excel pivot table in order to obtain a summary picture of the reported events and to plan further actions.

\section{Results}

In 2003, a total of 1189 errors were reported in 1151 reports; this number increased to 1669 errors in 2004. For 2003, this corresponded to a rate of 1 error for every 142 patients or 1 per 1223 tests. Most (81\%) of the errors were in the pre-analytical categories. Of the remainder, $10 \%$ were analytical, $8 \%$ were post-analytical, and $1 \%$ was service-related.

The majority of the events were due to human errors $(82.6 \%)$, with only a few $(4.3 \%)$ attributed to technical errors. Some events could not be classified and were labelled as unknown $(8.7 \%)$, whereas $4.5 \%$ were a mixture of several types of errors. The error rates per 100,000 tests for the years 2003-2004 are shown in Table 1.

The main type of error (47\%) involved samples sent to the laboratory (Table 1 "received") from general practitioners or hospital wards. At these sites, nontechnicians performed phlebotomy. The next two most frequent error types were in the categories of "Ordering" (15\%) and "Collection" (15\%), followed closely by "Interference" (10\%), due primarily to haemolysis (Table 1).

In 2003, a total of 89 incidents $(7.5 \%)$ involved patient misidentification. Only four of these errors were made within the laboratory, with the majority of these types of errors made by practitioners $(n=56)$ and wards $(n=25)$. Other clerical errors (Missing/ Wrong Order/Missing Physician/Ward ID) were fairly common among samples received from both practitioners $(n=28,2 \%)$ and wards $(n=123,10 \%)$. In 14 cases, samples were collected from the incorrect patient but labelled with the expected patient's identity. The clinicians noticing unexpected test results discovered most of these errors. Insufficient or failure to centrifuge samples was also a fairly frequent type of error $(n=48,4 \%)$ among the samples received. Haemolysis dominated the interference category in both 2003 (98/116) and 2004 (366/386). Approximately half of the samples came from practitioners, but a significant number came from the haematology, oncology, and dialysis wards where in vivo haemolysis could be expected. The reason for haemolysis could not be determined in most cases, although human error was suspected in roughly half of the cases. Some practitioners experienced several incidents with great fluctuation between the 2 years, whereas others had no cases at all. No systematic differences in the centrifugation or transportation route were observed between practitioners who had high vs. low frequencies of haemolysis.

\section{Discussion}

By the end of the first month, the pattern of error types that were seen throughout the first 2 years had 
Table 1 Error type and sub-error type per 100,000 performed tests.

\begin{tabular}{|c|c|c|c|}
\hline Error type & Sub-error type & 2003 & 2004 \\
\hline Pre-analytical & Total & $\mathrm{n}=959$ & $n=1178$ \\
\hline Ordering & $\begin{array}{l}\text { Sample without order; duplicate order; insufficient clinical } \\
\text { information; missing/incorrect patient ID; missing/incorrect } \\
\text { physician ID; incorrect test; test ordered on wrong patient } \\
\text { test; not readable; not fasting; incorrect time/date; incorrect } \\
\text { priority; typing error; other }\end{array}$ & $\begin{array}{l}12.7 \\
(n=184)\end{array}$ & $\begin{array}{l}15.6 \\
(n=248)\end{array}$ \\
\hline Collection & $\begin{array}{l}\text { Forgotten; failed; incorrect tube; lost tube; incorrect volume; } \\
\text { incorrect temperature; incorrect treatment; incorrect patient; } \\
\text { not fasting; incorrect time/date; incorrect priority; tube } \\
\text { missing/incorrect labelled; patient not present; other }\end{array}$ & $\begin{array}{l}12.0 \\
(n=174)\end{array}$ & $\begin{array}{l}12.7 \\
(n=202)\end{array}$ \\
\hline Distribution & $\begin{array}{l}\text { Forgotten; incorrect sorted; sent to wrong laboratory; lost; } \\
\text { incorrect packed; incorrect treatment; other }\end{array}$ & $\begin{array}{l}1.4 \\
(n=21)\end{array}$ & $\begin{array}{l}1.4 \\
(n=23)\end{array}$ \\
\hline Centrifugation & $\begin{array}{l}\text { Forgotten; incorrect speed/time; too late; tube broken/label } \\
\text { lost; incorrect temperature; insufficient centrifugation; other }\end{array}$ & $\begin{array}{l}0.8 \\
(n=12)\end{array}$ & $\begin{array}{l}1.4 \\
(n=23)\end{array}$ \\
\hline Pipetting & $\begin{array}{l}\text { Forgotten; missing/incorrect patient ID; Too late pipetting; } \\
\text { incorrect pipetting; other }\end{array}$ & $\begin{array}{l}0.8 \\
(n=11)\end{array}$ & $\begin{array}{l}1.0 \\
(n=16)\end{array}$ \\
\hline Freezing & $\begin{array}{l}\text { Forgotten; freezing delayed; incorrect temperature; lost; } \\
\text { other }\end{array}$ & $\begin{array}{l}0.5 \\
(n=7)\end{array}$ & $\begin{array}{l}0.2 \\
(n=3)\end{array}$ \\
\hline Received & $\begin{array}{l}\text { Not received; insufficient centrifugation; sample stability } \\
\text { exceeded; incorrect volume; missing/incorrect patient ID; } \\
\text { wrong tube; incorrect temperature; incorrect time/date; } \\
\text { cleaning swap in tube; insufficient clinical information; } \\
\text { tube missing/incorrect labelled; missing/incorrect physician } \\
\text { ID test; not readable; other }\end{array}$ & $\begin{array}{l}37.8 \\
(n=550)\end{array}$ & $\begin{array}{l}41.5 \\
(n=660)\end{array}$ \\
\hline Analytical & Total & $\mathrm{n}=123$ & $\mathrm{n}=397$ \\
\hline Interference & $\begin{array}{l}\text { Icterus; haemolysis; lipaemia; delayed coagulation; } \\
\text { coagulated; drugs; antibodies; other }\end{array}$ & $\begin{array}{l}8.0 \\
(n=116)\end{array}$ & $\begin{array}{l}24.3 \\
(n=386)\end{array}$ \\
\hline Reagent & $\begin{array}{l}\text { Incorrect reagent; expired reagent; incorrect stored; defect } \\
\text { reagent; incorrect dissolved reagent; other }\end{array}$ & $\begin{array}{l}0.0 \\
(n=0)\end{array}$ & $\begin{array}{l}0.2 \\
(n=3)\end{array}$ \\
\hline Apparatus & $\begin{array}{l}\text { Power failure; computer error; technical error; handling } \\
\text { error; periodic error; other }\end{array}$ & $\begin{array}{l}0.5 \\
(n=7)\end{array}$ & $\begin{array}{l}0.5 \\
(n=8)\end{array}$ \\
\hline Post-Analytical & Total & $\mathrm{n}=99$ & $\mathrm{n}=93$ \\
\hline Calculation & $\begin{array}{l}\text { Incorrect calculated; incorrect formula; decimal error; } \\
\text { dilution error; calibration error; other }\end{array}$ & $\begin{array}{l}0.3 \\
(n=5)\end{array}$ & $\begin{array}{l}0.7 \\
(n=11)\end{array}$ \\
\hline Procedure & $\begin{array}{l}\text { Internal control failed; external control failed; typing error; } \\
\text { incorrect released result; procedure not followed; other }\end{array}$ & $\begin{array}{l}4.7 \\
(n=69)\end{array}$ & $\begin{array}{l}3.9 \\
(n=62)\end{array}$ \\
\hline Reporting & $\begin{array}{l}\text { Typing error; decimal error; incorrect unit; procedure not } \\
\text { followed; result corrected; other }\end{array}$ & $\begin{array}{l}1.1 \\
(n=16)\end{array}$ & $\begin{array}{l}1.2 \\
(n=19)\end{array}$ \\
\hline Delivering & $\begin{array}{l}\text { Transmission error; LIS error; delay; result without patient } \\
\text { ID; other }\end{array}$ & $\begin{array}{l}0.6 \\
(n=9)\end{array}$ & $\begin{array}{l}0.1 \\
(n=1)\end{array}$ \\
\hline Service & Total & $\mathrm{n}=8$ & $\mathrm{n}=1$ \\
\hline Information intern & Misinformation; missing information; misunderstanding; other & $\begin{array}{l}0.3 \\
(n=4)\end{array}$ & $\begin{array}{l}0.0 \\
(\mathrm{n}=0)\end{array}$ \\
\hline Information extern & Misinformation; missing information; misunderstanding; other & $\begin{array}{l}0.2 \\
(n=3)\end{array}$ & $\begin{array}{l}0.1 \\
(n=1)\end{array}$ \\
\hline Service & Lack of flexibility; complaints; other & $\begin{array}{l}0.1 \\
(n=2)\end{array}$ & $\begin{array}{l}0.0 \\
(n=1)\end{array}$ \\
\hline Total errors & & 1189 & 1669 \\
\hline Total tests & & $1,454,251$ & $1,589,198$ \\
\hline
\end{tabular}

already become apparent. Most reports dealt with only a single event, but 32 reports involved two events, and two reports involved three events. As with all registration systems, it is important to note that the self-reported events represent the minimum number of errors, since many erratic events go undetected and are never reported.

Few, if any, departments in healthcare institutions provide as many services as the biochemistry laboratories, and this workload has steadily increased over the past years. During the last decade, the num- ber of tests performed in our laboratory increased by $\sim 10 \%$ annually. Thus, it is not surprising that errors occur. However, the largest number of errors arose from outside the laboratory, possibly as a result of a lack of focus and experience. The error rate of 1 in 142 patients, or 1 per 1223 tests, in this study is within the same order of magnitude as that reported for general laboratories $(4,5)$, and it is slightly higher than those reported for two clinical chemistry laboratories (10). The frequency was lower than that reported from "STAT" laboratory sections $(11,12)$. 
Pre-analytical errors accounted for $81 \%$ of the errors recorded in our database. Other authors have also reported a high proportion of pre-analytical errors (5). Similar to our results, Valenstein and Meier reported a frequency of $4.8 \%$ for ordering errors among outpatient requisitions from 660 laboratories enrolled in the College of American Pathologists QProbe program (13).

At the start of this study, we attempted to classify errors according to severity. However, this proved impossible because of difficulties in accurately assessing the effect of each event on patients. The most potentially serious error, misidentification, was a frequent example in both the present work and other studies; rates as high as 55 misidentification errors per million tests have been reported (14). The Danish identification system, with the unique CPR-NR, allowed us to identify many inconsistencies in identification, such as discrepancies between the patient ID number and name, or within the number itself through use of the validation cipher. This was most pronounced for manually, paper-ordered samples, and decreased over time as more samples were ordered electronically. We have observed a case in which the incorrect patient name and ID data were used for blood type tests. Another reoccurring event was mix-ups in the neonatal ward, as parents or neonatal staff had a tendency to remove wristbands they considered to be annoying to the children (7). All of these event types could have been lethal if undetected. However, in most cases, the laboratories were not given any information regarding the consequences of these errors. Apart from the potentially lethal consequences, errors still increase the use of resources. If an error is detected, samples must be re-collected, procedures are delayed, and time is wasted.

The errors in this study were rarely technical in nature. Laboratories have tried (with success) to reduce the frequency of technical errors by employing engineering developments. Automatic robots with barcode readers and electronic data transfer capabilities have been an important factor. Many analysers have automatic quality control and calibration features that eliminate the possibility of loading incorrect or out-dated reagents. Furthermore, biochemical laboratories have long used quality control methods, including detailed manuals and guidelines, for all methods and procedures.

Similar to the results of other studies, we observed a high frequency of interference that was primarily due to haemolysis (15). We classified haemolysis as an analytical event, since the condition interferes with the measurement of some analytes. Furthermore, it is impossible to discriminate between in vivo or in vitro haemolysis. Others have reported the prevalence of haemolysis as a subset of pre-analytical events $(39 \%-69 \%)(15,16)$. The most common reasons for haemolysis appear to include the incorrect handling of tubes, incorrect additives, the presence of a vacuum remaining in tubes, premature centrifugation, incorrect transport, or variations in tube batches (15-17).
Despite the use of numerous manuals and written procedures, over $80 \%$ of the errors were human errors. Only a few areas showed a decrease in the number of events. At the beginning of our registration period, we noticed many events associated with testing of cervical samples for Chlamydia DNA. In many cases, the tube contained the cleaning swab instead of the sampling rod, or was improperly closed resulting in leakage of the medium during transportation. Following direct contact with the doctors involved, the frequency of these events was reduced nearly to zero by the end of the study. In contrast, the number of samples received with other errors remained constant throughout the year. We attempted to instruct the hospital ward and general practitioners by appointing a laboratory technician as a consultant to provide instruction in sampling and handling procedures. As these attempts to reduce errors were largely unsuccessful and the staff at the laboratory felt that error reporting was meaningless, it was essential to focus on internal errors. Therefore, we ceased reporting errors made outside the laboratory in 2005. This unfortunately limits the strength of this study, especially as pre-analytical errors are the most frequent and constitute an important part of the total testing process (4). We therefore report only the errors that occurred during 2003-2004, a time period for which the recording system was uniform.

Our trial period may have been too brief to demonstrate a decrease in error frequency, especially when one considers that it took over a decade to achieve a $34 \%$ error reduction in a STAT laboratory (12). Plebani mentions in his review that the error rate has been decreasing steadily over time $(3,4)$. In our experience, it is very difficult to change the behaviour of personnel that are not trained in laboratory practice. This might be taken into consideration with the increased use of point-of-care analysis. As electronic test ordering became more widespread among general practitioners in 2005, we observed a particular decrease in identification errors (data not shown). Generally, technical solutions such as intelligent ordering systems and controls for patient ID should be employed wherever possible (7).

In conclusion, we recommend that every clinical laboratory record their errors in a structured manner, as suggested by Lippi et al. (6). A relational database is a useful tool for the registration and extraction of data, but the database structure should reflect the workflow of each individual laboratory, as suggested by Lippi et al. (6). Our template is free upon request.

\section{References}

1. Kalra J. Medical errors: impact on clinical laboratories and other critical areas. Clin Biochem 2004;37:1052-62.

2. Plebani M. Errors in clinical laboratories or errors in laboratory medicine? Clin Chem Lab Med 2006;44:750-9.

3. Plebani M. Errors in laboratory medicine and patient safety: the road ahead. Clin Chem Lab Med 2007;45:700-7. 
4. Plebani M. Exploring the iceberg of errors in laboratory medicine. Clin Chim Acta 2009;404:16-23.

5. Bonini P, Plebani M, Ceriotti F, Rubboli F. Errors in laboratory medicine. Clin Chem 2002;48:691-8.

6. Lippi G, Banfi G, Buttarello M, Ceriotti F, Daves M, Dolci $A$, et al. Recommendations for detection and management of unsuitable samples in clinical laboratories. Clin Chem Lab Med 2007;45:728-36.

7. Lippi G, Blanckaert N, Bonini P, Green S, Kitchen S, Palicka $\mathrm{V}$, et al. Causes, consequences, detection, and prevention of identification errors in laboratory diagnostics. Clin Chem Lab Med 2009;47:143-53.

8. Boone DJ. How can we make laboratory testing safer? Clin Chem Lab Med 2007;45:708-11.

9. Signori C, Ceriotti F, Sanna A, Plebani M, Messeri G, Ottomano $\mathrm{C}$, et al. Process and risk analysis to reduce errors in clinical laboratories. Clin Chem Lab Med 2007;45:742-8.

10. Lapworth R, Teal TK. Laboratory blunders revisited. Ann Clin Biochem 1994;31:78-84.

11. Plebani M, Carraro P. Mistakes in a stat laboratory: types and frequency. Clin Chem 1997;43:1348-51.
12. Carraro P, Plebani M. Errors in a stat laboratory: types and frequencies 10 years later. Clin Chem 2007;53:133842.

13. Valenstein P, Meier F. Outpatient order accuracy. A College of American Pathologists Q-Probes study of requisition order entry accuracy in 660 institutions. Arch Pathol Lab Med 1999;123:1145-50.

14. College of American Pathologists, Valenstein PN, Raab SS, Walsh MK. Identification errors involving clinical laboratories: a College of American Pathologists Q-Probes study of patient and specimen identification errors at 120 institutions. Arch Pathol Lab Med 2006;130:1106-13.

15. Lippi G, Blanckaert N, Bonini P, Green S, Kitchen S, Palicka $V$, et al. Haemolysis: an overview of the leading cause of unsuitable specimens in clinical laboratories. Clin Chem Lab Med 2008;46:764-72.

16. Lippi G, Guidi GC. Risk management in the preanalytical phase of laboratory testing. Clin Chem Lab Med 2007;45: 720-7.

17. Lippi G, Bassi A, Brocco G, Montagnana M, Salvagno GL, Guidi GC. Preanalytic error tracking in a laboratory medicine department: results of a 1-year experience. Clin Chem 2006;52:1442-3. 